\title{
The Benefits of Testosterone Therapy in Poor Ovarian Responders Undergoing In Vitro Fertilisation (IVF)
}

\author{
Authors: \\ *Petya Andreeva, ${ }^{1,2}$ Ivelina Oprova, ${ }^{1}$ Luboslava Valkova, ${ }^{1}$ Petya \\ Chaveeva, ${ }^{1}$ Ivanka Dimova, ${ }^{3}$ Atanas Shterev' \\ 1. Shterev Hospital, Sofia, Bulgaria \\ 2. South-West University, Blagoevgrad, Bulgaria \\ 3. Medical University, Sofia, Bulgaria \\ *Correspondence to andreivp@yahoo.com
}

Disclosure: The authors have declared no conflicts of interest.

Acknowledgements: The authors would like to thank Ms Biliana Tsvetkova for English language editing of this paper. The ethics committees of the hospital approved this study. All procedures performed were in accordance with the ethical standards. All participants provided written informed consent.

Received:

12.04 .20

Accepted:

02.07 .20

Keywords:

Cycle cancellation, in vitro fertilisation (IVF), poor ovarian responders (POR), pregnancy rate $(P R)$, testosterone.

Citation:

EMJ. 2020;5[3]:71-79.

\begin{abstract}
Introduction: Poor ovarian responders are the most challenging patients in reproductive medicine and no successful treatment has been proposed. Androgens are thought to play an important role during early folliculogenesis and diminished levels are associated with decreased ovarian sensitivity to follicle-stimulating hormone. This study aimed to determine whether pretreatment with testosterone improves the results in poor responders undergoing in vitro fertilisation (IVF).
\end{abstract}

Materials and methods: This observational pilot study enrolled 33 poor responders undergoing IVF. Eleven patients were pretreated with $250 \mathrm{mg}$ intramuscular testosterone and compared to a control group of 22 patients. The participants were tested for free testosterone, dehydroepiandrosterone sulfate, sex hormone binding globulin, and anti-mullerian hormone (AMH).

Results: The two groups had similar baseline characteristics. Significant improvement was reached in the hormones free testosterone, dehydroepiandrosterone sulfate, and sex hormone binding globulin in the testosterone-pretreatment group. No difference was detected in antral follicle count (5.06 versus 4.24); $\mathrm{AMH}$ (0.51 versus 0.53 ), mature oocytes (2.2 versus 2.32 ), and the number of embryos (1.2 versus 1.33) between the study and control groups, respectively. There was a slow improvement in fertilisation rate but without any significance (62.97\% versus 57.61\%). However, the cancellation rate of the ovarian stimulation was much greater in the control group (18.18\%) in comparison with the study group (0.0\%). Pregnancy rate (PR) in the testosterone group was higher than controls (PR per cycle: $27.3 \%$ versus $4.6 ; p=0.09$ ). 
Conclusion: Based on the limited number of patients studied, pretreatment with testosterone seems to improve PR and cancellation rate in poor responders but failed to affect antral follicle count, $A M H$, and the number of mature oocytes and embryos. Given these results, further research would provide more certainty.

\section{INTRODUCTION}

The most challenging patients for fertility care providers are the poor ovarian responders (POR). During the years, many attempts have been made to define the profile of POR but the most useful classifications remain the Bologna criteria 2011 and the later Patient-Oriented Strategies Encompassing IndividualizeD Oocyte Number (POSEIDON) criteria. ${ }^{1-5}$ In 2016, POSEIDON ${ }^{6}$ were developed, which are a more specific and more flexible prognosis concept for improving the management of patients undergoing assisted reproductive technologies. The proposed POSEIDON stratifies patients into four groups on the basis of age (over and under 35), antimullerian hormone (AMH) levels, and antral follicle count (AFC); Group 1 and 2 are defined as suboptimal responders with good prognosis and Group 3 and 4 as low responders with poor prognosis. This classification was created to serve as a guide to personalise treatment protocols and improve the management of lowprognosis patient to maximise in vitro fertilisation (IVF) success.

Reproductive plans are often delayed and the proportion of patients with POR has become massive. Different pharmacological approaches have been proposed to improve the outcomes of IVF treatment but none have been established with certainty. In recent decades, there has been an increased interest in the role of androgen supplementation while undergoing IVF. In 2000, Casson et al. first suggested that patients with POR could benefit from androgens. ${ }^{7}$ Thereafter, a series of studies of patients with POR reported that dehydroepiandrosterone (DHEA) supplementation not only improves oocyte yield but also positively affects the egg and embryo quality and IVF pregnancy rate $(P R)^{8-17}$

However, recent large-scale randomised trials have failed to confirm this, and highlight free testosterone $(T)$ levels as a more appropriate androgen in the improvement of the ovarian reserve. ${ }^{18,19}$ It has been established that there is a direct $T$ effect on the ovaries through androgen receptors (AR), and sufficient AR are necessary for normal follicular development and function. ${ }^{17,18}$

Granulosa cells (GC) express AR at early stages of folliculogenesis, with these stages of follicle maturation occurring months before ovulation. Given this, it would be logical to assume that longer androgen supplementation would be associated with better results for follicular activation and recruitment, and thus reach a larger pool of gonadotrophin-sensitive follicles and respectively more oocytes, which appear to improve reproductive outcomes. ${ }^{19,20}$

Certain modalities have been tested through different studies and trials. In daily practice, the most commonly used $T$ agents, before or during ovarian stimulation, are transdermal testosterone. Some meta-analyses evaluated the effect of different doses or duration of treatment, but the most common duration of therapy was 21 days. ${ }^{19,20}$ The physiological cycle of the transition of primordial to preantral follicles lasts for 290 days, but in this process the levels of expressed $A R$ are low. However in the preantral stage of follicular development, androgens, through a synergistic interaction between nuclear and extranuclear signalling, induce the expression of AR in GC, contributing to follicular survival and growth. Simultaneously, this increases folliclestimulating hormone (FSH) receptors (FSHR) that enhance the sensitivity of preantral follicles toward FSH actions. These androgen-AR actions together promote preantral follicle growth and transition to antral stage, taking at least 65 days (Figure 1)..$^{21,22}$

Beyond the above-mentioned data, this study aimed to investigate the effects of testosterone pretreatment on the ovarian reserve and IVF outcome in patients with POR but with longer exposition and higher dosage of testosterone administered intramuscularly. 

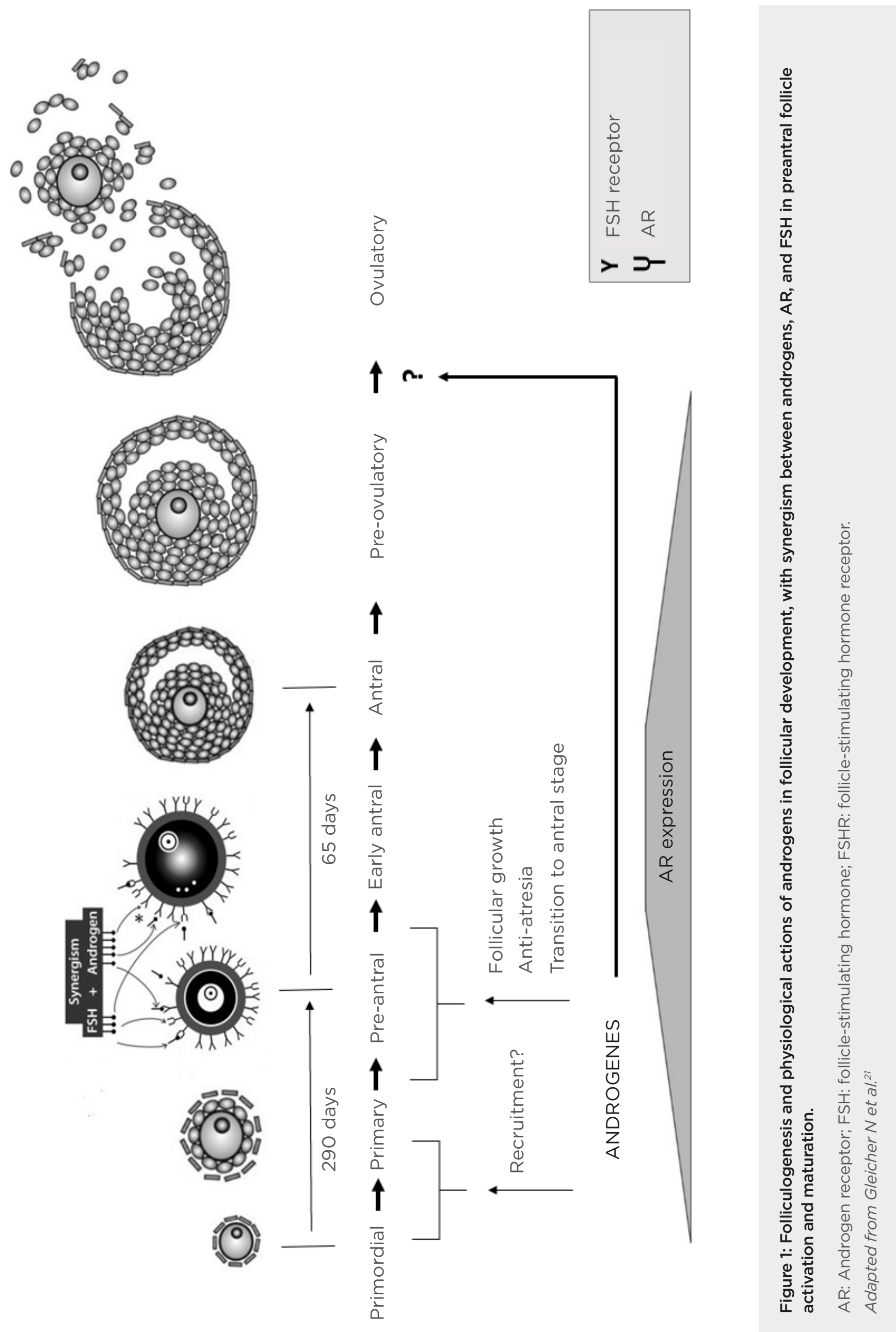


\section{MATERIALS AND METHODS}

An observational pilot study was performed at the IVF department of a private obstetrics and gynaecology hospital. The inclusion criteria were female patients with POR who had at least one previous failed or cancelled IVF cycle and met criteria for POSEIDON Group 4.5,6 The patients in the study had a mean age of 39.4 years, mean AMH levels of $0.52 \mathrm{ng} / \mathrm{mL}, A F C<5$, number of retrieved oocytes $<5$, and level of oestradiol on the day of human chorionic gonadotropin therapy $<1,200 \mathrm{pg} / \mathrm{mL}$. No significant difference was observed in demographic and baseline characteristics between the two groups. Exclusion criteria were uterine malformations, hydrosalpinx, $\mathrm{FSH}>2 \mathrm{O} \mathrm{IU} / \mathrm{L}$, or severe male factor.

\section{Pretreatment with Testosterone in the Study Group}

In all eligible patients, following signed informed consent, $250 \mathrm{mg}$ of intramuscular testosterone was administered twice for 6 weeks. The active substances in the injections were testosterone propionate $30 \mathrm{mg}$, testosterone phenylpropionate $60 \mathrm{mg}$, testosterone isocaproate $60 \mathrm{mg}$, and testosterone decanoate $100 \mathrm{mg}$.

\section{Ovarian Stimulation Protocol}

IVF embryo transfer procedure was performed in all patients. A gonadotropin-releasing hormone antagonist protocol was used for the ovarian stimulation. After confirming baseline blood levels and excluding any functional ovarian cysts, ovarian stimulation with follitropin alfa was started on Day 2 of the menstrual cycle and 4 weeks after the last testosterone application. Patients in the control group underwent the same protocol, without receiving testosterone pretreatment. During ovarian stimulation, regular transvaginal ultrasound scanning and monitoring of serum concentrations of luteinising hormone, oestradiol, and progesterone were undertaken. Ovum pick-up was performed 34-36 hours after the subcutaneous administration of 5,0006,500 IU human chorionic gonadotropin for the ovulation trigger, and the matured oocytes were fertilised by an intracytoplasmic sperm injection. All embryos were transferred at the cleavage or blastocyst stage. Luteal support was performed via transvaginal administration of progesterone, starting on the day of ovum pick-up.

\section{Hormonal Measurements}

All patients were tested for $T$ (nmol/L), DHEA sulphate (DHEA-S) $(\mu \mathrm{mol} / \mathrm{L})$, sex hormone binding globulin (SHBG) ( $\mathrm{nmol} / \mathrm{L})$, and $\mathrm{AMH}$ (ng/ $\mathrm{mL}$ ). Patients eligible for $\mathrm{T}$ treatment were those with SHBG $<80 \mathrm{nmol} / \mathrm{L}$, T less than one-third of the normal range, and DHEA-S within the normal range. All hormones were analysed before and after $\mathrm{T}$ treatment in the tested group.

\section{Outcome Measures}

The primary outcome measure was the PR. The secondary outcome measures included the number of antral follicles, developing embryos, and mature (M2) oocytes (COC); fertilisation rate (FR); and cancellation rate (CR). Additionally, changes in the serum hormonal levels of $A M H, T$, SHBG, and DHEA-S were observed.

FR was calculated by dividing the number of fertilised oocytes by the number of M2 oocytes. Embryo quality was assessed according to morphological criteria based on the assessment of the blastomeres and the degree of blastomere fragmentation. Clinical pregnancy was defined as the presence of an intrauterine sac with fetal heart palpitation.

\section{Sample Size}

The above-described criteria were observed for the selection of patients who were able to receive testosterone therapy. From all tested patients, 33 met eligibility criteria and, after obtaining their informed consent, 11 patients were enrolled in the T pretreatment group and 22 in the control group.

\section{Statistical Analysis}

Statistical analysis was performed. The odds ratio, with its standard error and 95\% confidence interval, were calculated. For test of significance, the $p$ value was calculated and statistical significance was considered at $\mathrm{p}<0.05$. All analyses were performed with MedCalc ${ }^{\circledR}$ statistical software.

\section{RESULTS}

All 33 patients were divided into two groups: the study group of 11 participants received pretreatment with $250 \mathrm{mg}$ intramuscular testosterone twice, every $3^{\text {rd }}$ week and the other 22 participants formed the control group without pretreatment. 
Table 1: The main results in the testosterone-pretreatment group and in the control group.

\begin{tabular}{|c|c|c|c|c|}
\hline & T-pretreatment group & Control group & $\begin{array}{c}\text { OR } \\
(95 \% \mathrm{Cl})\end{array}$ & $p$ value \\
\hline AFC & 5.06 & 4.24 & & $p=0.4$ \\
\hline $\mathrm{AMH}(\mathrm{ng} / \mathrm{mL})$ & 0.51 & 0.53 & & $p=0.9$ \\
\hline $\begin{array}{c}\text { COC } \\
\text { (M2 oocytes) }\end{array}$ & 2.20 & 2.32 & & $p=0.9$ \\
\hline $\begin{array}{l}\text { Total number of } \\
\text { embryos }\end{array}$ & 1.20 & 1.33 & & $p=0.6$ \\
\hline $\begin{array}{l}\text { Mean number of } \\
\text { transferred embryos }\end{array}$ & 1.33 & 1.05 & & $p=0.5$ \\
\hline FR (\%) & 62.97 & 57.61 & 1.32 & $p=0.6$ \\
\hline CR (\%) & 0 & 18.20 & 5.60 & $p=0.042$ \\
\hline PR (\%) & 27.30 & 4.60 & 7.88 & $p=0.093$ \\
\hline SHBG $(n m o l / L)$ & 57.44 & 104.77 & & $p=0.02$ \\
\hline $\mathrm{T}(\mathrm{nmol} / \mathrm{L})$ & 28.97 & 0.99 & & $p<0.0001$ \\
\hline DHEA-S $(\mu \mathrm{mol} / \mathrm{L})$ & 10.13 & 5.29 & & $p=0.0086$ \\
\hline
\end{tabular}

AFC: antral follicle count; AMH: anti-mullerian hormone; $\mathrm{Cl}$ : confidence interval; COC: cumulus-oocyte complex; CR: cancellation rate; DHEA-S: dehydroepiandrosterone sulfate; FR: fertilisation rate; OR: odds ratio; PR: pregnancy rate; SHBG: sex hormone binding globulin; T: free testosterone.

The main demographic and baseline characteristics of all patients were very similar, including age, BMI, causes and duration of infertility, AFC, DHEA-S, T, SHBG, and AMH, with a mean $\mathrm{BMI}$ of 25.6 and mean age of 39.6 years. A significant improvement was reached in the levels of T, DHEA-S, and SHBG in the T pretreatment group (Table 1). No differences were detected in the AFC between the groups (5.06 versus 4.24), $\mathrm{AMH}$ (0.51 versus 0.53 ), number of M2 oocytes retrieved (2.20 versus 2.32 ), and the total number of embryos (1.20 versus 1.33) (Table 1). The analysis showed a slow improvement of the FR in the study group but without a significance (62.97\% versus $57.61 \%$, respectively; $p=0.6$ ). However, the CR of the ovarian stimulation in one cycle was much larger in the control group in comparison with the study group (18.8\% versus $0.0 \% ; \mathrm{p}<0.05)$. The results showed an almost eight-fold increase in the odds of pregnancy after T treatment in comparison with no pretreatment; PR per cycle in the interventional group was $27.3 \%$ and $4.6 \%$ in the control group, with odds ratio 7.88 in $95 \%$ confidence interval $(p=0.09)$ (Table 1 and Figure 2).

\section{DISCUSSION}

There is evidence that testosterone levels in individuals with diminished ovarian reserve decrease significantly, irrespective of age or premature ovarian ageing. ${ }^{23,24}$ 


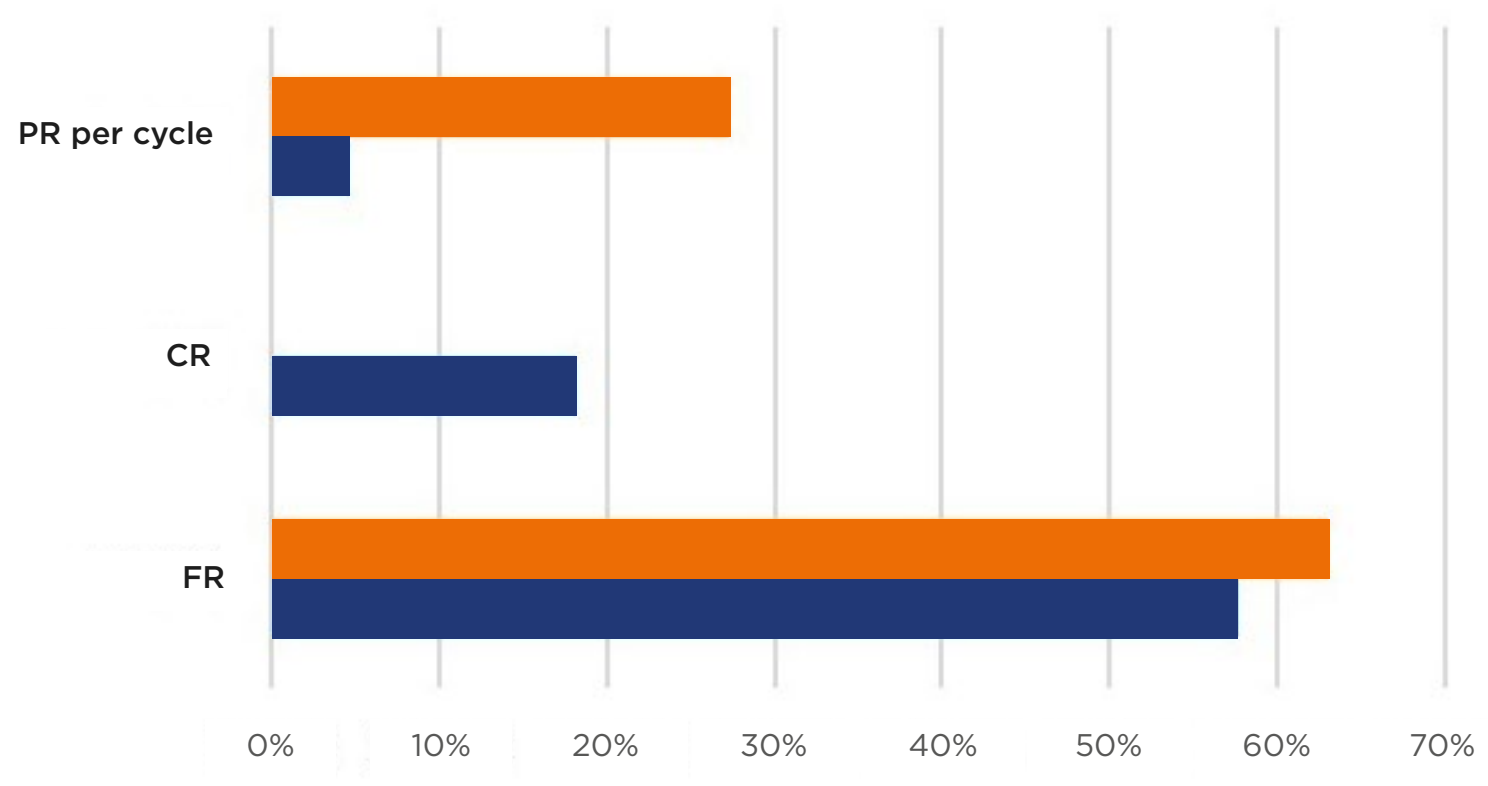

Testosterone pretreatment group

Control group

Figure 2: Pregnancy rate, cancellation rate, and fertilisation rate results.

CR: cancellation rate; FR: fertilisation rate; PR: pregnancy rate.

According to the 'Two cell, two gonadotropin' theory of ovarian steroidogenesis, oestrogen's synthesis in GC originates from androgens from thecal cells. Over the years, understanding of the effect of androgens on the ovary process and female fertility has developed significantly. Androgens are considered to be detrimental to ovarian function, and sufficient androgen actions through the AR are necessary for normal follicle development and function. ${ }^{21,25}$ AR expression can be found in GC of mostly immature preantral and early-antral follicles; thereafter, they decrease with advancing follicle maturation, suggesting the importance of androgens mainly in the early stages of follicle maturation 21,22,26 (Figure 1). Many studies demonstrate androgens as essential for follicular recruitment, follicular growth, and reduction in GC apoptosis leading to an increase in the number of growing follicles. ${ }^{27-29}$ Additionally, AR actions induce the expression of the micro-RNA miR-125b that decreases pro-apoptotic proteins. It has been proposed that in the ovary androgens maintain a certain level of miR-125b expression, essential for the balance between follicular survival and atresia. ${ }^{30}$
Furthermore, androgens have been found to induce FSHR mRNA expression during preantral to antral follicle progression, whether this induction by androgens is mediated through androgen-AR response or by direct synergism between androgens and FSH in the ovary. This suggests that androgen stimulation enhances follicular sensitivity toward FSH actions by increasing FSHR levels, which potentially contributes to follicle growth (Figure 1).31-36

It is known that androgens, acting via the AR, may also regulate the expression and action of key ovarian growth factors during different stages of follicle growth, which indicates that an intraovarian growth factor system plays an essential role in ovarian follicular development, regulated by androgen-AR actions. ${ }^{37}$ A study demonstrated that during the ovulation process the androgen-AR pathway also plays a role in the last stage of folliculogenesis. Androgens produced by the luteinising hormone surge are likely to act through the AR and may be involved in the ovulatory process by directly regulating the expression of COX2 and AREG genes and their actions (Figure 1). ${ }^{38}$ 
Taking into consideration the above-mentioned evidence, long pretreatment with testosterone could be beneficial for ovarian response. Despite the significantly higher testosterone level after 6 weeks of administration compared with no pretreatment, no statistically significant changes were detected in this study in the mean number of AFC (5.06 versus 4.24, respectively), mature oocytes retrieved (2.2 versus 2.32 ), and total number of embryos (1.2 versus 1.33) (Table 1). In agreement with this study, Massin et al. ${ }^{39}$ performed a randomised controlled trial with 49 patients and found a nonsignificant increase with testosterone pretreatment $(10 \mathrm{mg} /$ day for 15-21 days) on the number of COC. Six years later, a systematic review and meta-analysis was performed by Gonzalez-Comadran et al. ${ }^{40}$ and no differences were observed regarding the number and quality of the oocytes retrieved. In 2016, 26 patients were randomly pretreated with $\mathrm{T}$, but it failed to increase the COC. ${ }^{41}$ Contrary to this, Noventa et al. ${ }^{42}$ observed that a higher number of total oocytes, M2 oocytes, and total embryos were developed after $\mathrm{T}$ therapy. The same was shown by Kim et al., ${ }^{43,44}$ in a significant improvement in the number of COC retrieved, and showing that the level of success is time dependent (COC retrieved with pretreatment for 2 weeks: 4.3 versus 1.6; for 3 weeks: 5.3 versus 2.0; and for 4 weeks: 5.8 versus 1.9). These inconsistent results highlight the possibility that the evaluated studies might differ by the type of substance, the timing and the duration of the treatment, and by the mechanism of action.

Apart from the idea that androgens may regulate $\mathrm{AMH}$ and that some studies show a possible positive relationship between androgens and $\mathrm{AMH}$ levels in follicular fluid, the direct evidence of androgen-induced $\mathrm{AMH}$ expression and its underlying mechanism in GC are still lacking. ${ }^{45}$ The same result was observed in this study where no difference in serum AMH was observed after $\mathrm{T}$ therapy ( 0.51 versus 0.53 ). Vuong $\mathrm{LN}$ et al. ${ }^{46}$ performed long-term intraovarian androgen priming but also did not find any significant effect on AMH level. However, despite androgens improving recruitment and activation of prenatal follicles and the fact that $A M H$ is synthesised in $\mathrm{GC}$, there are insufficient studies observing the effect of testosterone on AMH.

This study revealed a significant improvement in the $\mathrm{CR}$ of ovarian stimulation in the $\mathrm{T}$ group
( $0.0 \%$ versus $18.2 \%$ in the control group; $\mathrm{p}<0.05$ ). This is important in impacting time, money, and patients' hope. This effect of testosterone could be explained as a result of the increased levels of FSHR mRNA in GC after androgen supplementation and supports the fact that androgens enhance follicle responsiveness to FSH, particularly in early antral stages, and the theory of a synergistic effect of androgens with FSH over folliculogenesis. ${ }^{32,34}$

Despite these conflicting results, the vast majority of studies claimed greater clinical PR after testosterone therapy, consistent with this study's results (PR per cycle: $27.3 \%$ in T group versus $4.6 \%$ in control group; $p=0.09$, close to significance). Noventa et al. ${ }^{42}$ performed a metaanalysis of available randomised controlled trials on the effect of transdermal testosterone, demonstrating a higher clinical PR and live birth rate. Recently, Vishwakarma et al. ${ }^{47}$ reported an improvement in the numbers of cryopreserved embryos per cycle and hence the cumulative PR.

\section{Limitation and Strengths of the Study}

This observational pilot study has some potential limitations that need to be considered; mainly, the relatively small number of patients included. This low rate of events decreases confidence in the results. Secondly, there are no other trials with intramuscular application of testosterone, so comparison was made with other types of administration which could give some bias. However, this limitation simultaneously could be counted as a strength of the study, because it is the first report of this type. In the literature, the most common route of administration was transdermal and only one other study reported an alternative method: intraovarian priming. ${ }^{46}$

\section{CONCLUSION}

Long-term testosterone pretreatment could be considered promising in IVF treatment of patients with POR. The findings of this study show improvement of the $P R$ and $C R$. No effect was observed on AFC, AMH, M2 oocytes retrieved, and the total number of embryos. Due to the limitations described above, more studies should be performed with a larger population and better adjustment with ovarian physiology by dose, timing, type, and duration of the testosterone therapy. 


\section{References}

1. Kailasan C et al. Defining poor ovarian response during IVF cycles, in women aged $<40$ years and its relationship with treatment outcome. Hum Reprod. 2004;19(7):1544-7.

2. Arslan M. Controlled ovarian hyperstimulation protocols for in vitro fertilization: two decades of experience after the birth of Elizabeth Carr. Fertil Steril. 2005;84(3):555-69.

3. Sallam $\mathrm{HN}$ et al. Defining poor responders in assisted reproduction. Int J Fertil Womens Med. 2005;50(3):115-20.

4. Turhan NO. Poor response-the devil is in the definition. Fertil Steril. 2006;85(4):e1.

5. Ferraretti AP et al; ESHRE working group on Poor Ovarian Response Definition. ESHRE consensus on the definition of 'poor response' to ovarian stimulation for in vitro fertilization: the Bologna criteria. Hum Reprod. 2011;26(7):1616-24.

6. Alviggi $C$ et al. A new more detailed stratification of low responders to ovarian stimulation: from a poor ovarian response to a low prognosis concept. Fertil Steril. 2016;105(6):1452-3.

7. Casson PR et al. Dehydroepiandrosterone supplementation augments ovarian stimulation in poor responders: a case series. Human Reprod. 2000;15(10):2129-32.

8. Barad DH, Gleicher N. Increased oocyte production after treatment with dehydroepiandrosterone. Fertil Steril. 2005;84(3):756.

9. Barad DH, Gleicher N. Effect of dehydroepiandrosterone on oocyte and embryo yields, embryo grade and cell number in IVF. Hum Reprod. 2006;21(11):2845-9.

10. Gleicher N, Barad DH. Effects of transdermal testosterone application on the ovarian response to FSH in poor responders undergoing assisted reproduction technique - a prospective, randomized, double-blind study. Hum Reprod. 2006;21(11):3027

11. Gleicher N, Barad DH. Dehydroepiandrosterone (DHEA) supplementation in diminished ovarian reserve (DOR). Reprod Biol Endocrinol. 2011;9:67.

12. Mamas L, Mamas E. Premature ovarian failure and dehydroepiandrosterone. Fertil Steril. 2009;91(2):644-6

13. Sonmezer M et al. Dehydroepiandrosterone supplementation improves ovarian response and cycle outcome in poor responders. Reprod Biomed Online. 2009;19(4):508-13.
14. Sunkara SK et al. Should androgen supplementation be used for poor ovarian response in IVF? Hum Reprod. 2012;27(3):637-40.

15. Hyman JH et al. DHEA supplementation may improve IVF outcome in poor responders: a proposed mechanism. Eur J Obstet Gynecol Reprod Biol. 2013;168(1):49-53.

16. Yilmaz $\mathrm{N}$ et al. Dehydroepiandrosterone supplementation improves predictive markers for diminished ovarian reserve: serum $\mathrm{AMH}$, inhibin $\mathrm{B}$ and antral follicle count. Eur J Obstet Gynecol Reprod Biol. 2013;169(2):257-60.

17. Yeung TW et al. A randomized, controlled, pilot trial on the effect of dehydroepiandrosterone on ovarian response markers, ovarian response, and in vitro fertilization outcomes in poor responders. Fertil Steril. 2014;102(1):108-15.e1.

18. Polyzos NP et al; T-TRANSPORT Investigators Group. Testosterone for poor ovarian responders: lessons from ovarian physiology. Reprod Sci. 2018;25(7):980-2.

19. Sunkara SK, Coomarasamy A. Androgen pretreatment in poor responders undergoing controlled ovarian stimulation and in vitro fertilization treatment. Fertil Steril. 2011;95(8):e73-4.

20. Bosdou JK. et al. The use of androgens or androgen-modulating agents in poor responders undergoing in vitro fertilization: a systematic review and meta-analysis. Hum Reprod Update. 2012;18(2):127-45.

21. Gleicher $\mathrm{N}$ et al. The role of androgens in follicle maturation and ovulation induction: friend or foe of infertility treatment? Reprod Biol Endocrinol. 2011:9:116.

22. Prizant et al. Androgen actions in the ovary: balance is key. J Endocrinol. 2014;222(3):R141-51.

23. Gleicher $\mathrm{N}$ et al. Hypoandrogenism in association with diminished functional ovarian reserve. Hum Reprod. 2013;28(4):1084-91.

24. Barbieri RL et al. Association of body mass index, age, and cigarette smoking with serum testosterone levels in cycling women undergoing in vitro fertilization. Fertil Steril. 2005;83(2):302-8

25. Fanchin R et al. Androgens and poor responders: are we ready to take the plunge into clinical therapy? Fertil Steril. 2011;96(5):1062-5

26. Vendola $\mathrm{K}$ et al. Androgens promote oocyte insulin-like growth factor I expression and initiation of follicle development in the primate ovary. Biol Reprod. 1999;61(2):353-7.

27. Mori T et al. Evidence for androgen participation in induced ovulation in immature rats. Endocrinology. 1977;101(2):623-6.
28. Ware VC. The role of androgens in follicular development in the ovary. I. A quantitative analysis of oocyte ovulation. J Exper Zool. 1982;222(2):155-67.

29. Wang $\mathrm{H}$ et al. Effect of adrenal and ovarian androgens on type 4 follicles unresponsive to FSH in immature mice. Endocrinology. 2001:142(11):4930-6

30. Sen A et al. Androgens regulate ovarian follicular development by increasing follicle stimulating hormone receptor and microRNA$125 \mathrm{~b}$ expression. Proc Natl Acad Sci U S A. 2014;111(8):3008-13.

31. Vendola KA et al. Androgens stimulate early stages of follicular growth in the primate ovary. J Clin Invest. 1998;101(12):2622-9.

32. Weil SJ et al. Androgen and folliclestimulating hormone interactions in primate ovarian follicle development. $\mathrm{J}$ Clin Endocrinol Metab. 1999;84(8):2951-6

33. Weil SJ et al. Androgen receptor gene expression in the primate ovary: cellular localization, regulation, and functional correlations. J Clin Endocrinol Metab. 1998;83(7):2479-85.

34. Nielsen ME et al. In human granulosa cells from small antral follicles, androgen receptor mRNA and androgen levels in follicular fluid correlate with $\mathrm{FSH}$ receptor mRNA. Molecul Hum Reprod. 2011;17(1):63-70.

35. Cardenas $\mathrm{H}$ et al. Androgen receptor and follicle-stimulating hormone receptor in the pig ovary during the follicular phase of the estrous cycle. Molecul Reprod Devel. 2002;62(1):92-8.

36. Luo W, Wiltbank MC. Distinct regulation by steroids of messenger RNAs for FSHR and CYP19A1 in bovine granulosa cells. Biology of Reproduction. 2006;75(2):217-25.

37. Orisaka M et al. Growth differentiation factor 9 promotes rat preantral follicle growth by up-regulating follicular androgen biosynthesis. Endocrinology. 2009;150(6):2740-8.

38. Yazawa T et al. Androgen/androgen receptor pathway regulates expression of the genes for cyclooxygenase- 2 and amphiregulin in periovulatory granulosa cells. Mol Cell Endocrinol. 2013;369(1-2):42-51.

39. Massin $\mathrm{N}$ et al. Effects of transdermal testosterone application on the ovarian response to $\mathrm{FSH}$ in poor responders undergoing assisted reproduction technique-a prospective, randomized, double-blind study. Hum Reprod 2006;21(5):1204-11.

40. Gonzalez-Comadran M et al. Effects of transdermal testosterone in poor responders undergoing IVF: systematic review and meta- 
analysis. Reprod Biomed Online. 2012;25(5):450-9.

41. Bosdou JK et al. Transderma testosterone pretreatment in poor responders undergoing ICSI: a randomized clinical trial. Hum Reprod. 2016;31(5):977-85.

42. Noventa $M$ et al. Testosterone therapy for women with poor ovarian response undergoing IVF: a metaanalysis of randomized controlled trials. J Assist Reprod Genet. 2019;36(4):673-83.
43. Kim CH et al. The effect of transdermal testosterone gel pretreatment on controlled ovarian stimulation and IVF outcome in low responders. Fertil Steril. 2011;95(2):679-83.

44. Kim CH et al. Ovarian features after 2 weeks, 3 weeks and 4 weeks transdermal testosterone gel treatment and their associated effect on IVF outcomes in poor responders. Dev Reprod. 2014;18(3):145-52.

45. Lebbe M, Woodruff TK. Involvement of androgens in ovarian health and disease. Mol Hum Reprod. 2013;19(12):828-37.

46. Vuong $L N$ et al. The effect of intraovarian androgen priming on ovarian reserve parameters in Bologna poor responders. Reprod Biomed Online. 2020;40(2):223-8.

47. Vishwakarma $\mathrm{P}$ et al. Role of testosterone pretreatment in poor ovarian responders undergoing in vitro fertilization/intracytoplasmic injection in comparison with growth hormone. IVF Life. 2016:3(3):90-7. 